\title{
A unifying model for concentration polarization, gel-layer \\ formation and particle deposition in cross-flow membrane filtration of colloidal suspensions
}

\author{
P. Bacchin ${ }^{*}$, D. Si-Hassen ${ }^{\ddagger}$, V. Starov ${ }^{\dagger}$, M.J. Clifton ${ }^{\ddagger}$, P. Aimar ${ }^{\ddagger}$ \\ ${ }^{\ddagger}$ Laboratoire de Génie Chimique, Université Paul Sabatier, 118 route de Narbonne, \\ 31062 Toulouse cedex, France \\ ${ }^{\dagger}$ Department of Chemical Engineering, Loughborough University, \\ Loughborough, Leicestershire, LE11 3TU, UK
}

*Corresponding author. bacchin@lgc.ups-tlse.fr, Fax number : (+33) 561556139

A model is proposed to describe the cross flow filtration of colloidal particles and molecules. This two-dimensional model depicts both concentration polarization and gel or cake formation in a tubular filtration device. A description of transport phenomena in a concentrated colloidal suspension is the core of the model. Surface and hydrodynamic interactions are used to predict the variation of the osmotic pressure and diffusion coefficient with the volume fraction of the suspension. The mathematical development leads to an analytical equation used for calculating the stationary permeate flux from integral calculations. The two-dimensional concentration profile along the membrane, together with the corresponding permeate flux is obtained. This paper illustrates how mass transfer equations coupled with a realistic description of the fluid can describe both concentration polarization and gel or 
cake formation. The paper includes a discussion on the differences between limiting and critical fluxes, and between particles and macromolecular cross flow filtrations.

Key words: filtration, fouling, modeling, membranes, colloids, ultrafiltration

\section{INTRODUCTION}

Cross-flow ultrafiltration is now used in a wide range of industrial applications (in the food industry, biotechnology, the pharmaceutical industry, water and waste-water treatment). The nominal molecular-weight cut-off of ultrafiltration membranes (1 kDa - $300 \mathrm{kDa})$ makes them appropriate for processing colloidal suspensions (particles or macromolecules whose size is less than $1 \mu \mathrm{m})$. Such a process is mainly limited by the accumulation of matter on the filter that includes concentration polarization and membrane fouling (formation of a gel layer or a deposit). With this in mind, the study of membrane fouling by colloidal dispersions is of considerable interest for developing the process. Here modeling opens up the possibility of better understanding mechanisms that reduce process efficiency, of optimizing the way the filtration should be operated and of creating expert systems for the design of membrane modules and membrane plants processing colloidal suspensions.

The complexity of colloidal matter comes from the presence of surface interaction between the suspended materials. Over the past two decades, experimental observations have revealed the role that colloidal interactions can play in the filtration of colloidal suspensions (Cohen \& Probstein, 1986; McDonogh, Fane \& Fell, 1989). Fifteen years ago the existing models were incapable of quantitatively predicting permeate flux and of qualitatively representing the effect of a suspension's physico-chemical properties, such as ionic strength or $\mathrm{pH}$, on the permeate flux. What was described by Cohen \& Probstein (1986) as "a colloid flux paradox" has recently been underlined by the experimental finding of a critical flux for colloids. This critical 
phenomenon demonstrates the specificity of the filtration of colloidal suspensions and raises interest for modeling in this area: just a small variation in operating conditions (particle size or surface charge, $\mathrm{pH}$, ionic strength, concentration, pressure, cross flow velocity, permeation rate, ...) induces important changes in the working point and so in the way the process has to be operated. As detailed in the next section, various models accounting for colloidal interaction have been recently developed to describe colloid filtration. However, the model for a limiting phenomenon in ultrafiltration is often selected according to the application (concentration polarization and gel layer for macromolecules, deposit for particles) and the models differ in their theoretical treatment. But colloidal suspensions often exhibit the behavior both of particles and of macromolecules, thus leading to a delicate choice as to the way modeling should be developed. Furthermore, detailed analysis of the effect of colloidal interaction on filtration is often carried out in a one-dimensional system (i.e. normal to the membrane surface), whereas the design of membrane modules and the definition of appropriate operating conditions has to take account of the development of the mass-transfer boundary layer. For the filtration of colloidal suspensions, this cannot be done using the standard calculation based on purely diffusive mechanisms, as was pointed out by Jönsson \& Jönsson (1996). However these authors suggested using an experimental technique to determine the boundary layer thickness; we shall show below how this thickness can be estimated from a two-dimensional model.

In the present work, we have investigated the possibility of introducing particle-particle colloidal interactions into a two dimensional analysis of transport phenomena along the length of a filtration device. This allows the specificity of colloid filtration to be accounted for. Also, a phase transition, related to the balance between dispersive and attractive forces, accounts for the passage from the liquid state to the gelled phase. By integrating such phenomena into the description of membrane fouling, the model can depict mechanisms of concentration polarization, gel formation and particle deposition, within a single approach. Important 
suggestions as to the way filtration should be operated when processing colloidal suspensions are underlined.

\section{BACKGROUND}

Solute accumulation is the antagonistic phenomenon of the filtration process and as such can only be partially reduced (for example by changing the hydrodynamics) but never totally eliminated. Solute accumulation is a self-regulating phenomenon as it causes a drop in permeate flux, thus inducing a simultaneous decrease in the accumulation rate and so on. Consequently, when operating at a fixed trans-membrane pressure difference (TMP) and feed concentration, surface fouling leads to a stationary or quasi-stationary permeate flux. This implies that two kinds of transfer are involved in fouling:

- solvent transfer (permeate flux) through the fouling layer, when present, and through the membrane,

- solute transfer (mass flux) to the membrane or to the fouling layer (if fouling has already occurred).

The solute transfer differs from the transfer directly induced by solvent convection because of the membrane's retention properties; this difference causes the solute accumulation. Physically, these two fluxes are intimately linked together and therefore have to be treated simultaneously in the theory: the solvent transfer is dependent on solute transfer as solute accumulation changes the osmotic pressure difference and, when relevant the hydraulic resistance of the fouling layer, while the mass transfer depends on solvent transfer as permeate flux is at the origin of mass flux. This study is restricted to surface solute accumulation, namely: concentration polarization, gel formation or particle deposition mechanisms. These phenomena are considered at steady state.

\section{Solvent transfer through the membrane and the fouling layer}

The starting point for describing solvent transfer in cake-filtration or osmotic-pressure models 
is the initial driving force: the transmembrane pressure. The cake-filtration model assumes that a layer of deposited matter is formed on the membrane, so the frictional drag due to permeation through these immobile particles leads to an additional hydraulic resistance that corrects the original Darcy law. The osmotic-pressure model considers that as there is a great concentration difference between the two sides of the membrane, a thermodynamic force arises in opposition to the TMP; this is equal to the transmembrane osmotic pressure. Combining both mechanisms, for a given transmembrane pressure $\Delta P$ and a hydraulic resistance of the clean membrane $R_{m}$, the permeate flux $V_{w}$ is given by:

$V_{w}=\frac{\Delta P-\Delta \Pi}{\mu\left(R_{m}+R_{c}\right)}$

where the two unknown parameters are the hydraulic resistance of the cake $R_{c}$ and the transmembrane osmotic pressure difference $\Delta \Pi$ across the cake and the membrane whose determination can only come from an accurate description of mass transfer near the membrane.

\section{Mass transfer to the membrane}

The convection-diffusion equation is widely used to describe the stationary permeate flux during filtration of small-size solutes at low concentration. For a totally retentive membrane, solute flux to the membrane is zero at steady state:

$$
-V_{w} \phi-D \frac{\partial \phi}{\partial z}=0
$$

After integration across the mass-transfer boundary layer, assuming a constant diffusion coefficient, the following relationship is found between the permeate flux and the membrane-tobulk concentration ratio:

$$
V_{w}=\frac{D}{\delta} \ln \left(\frac{\phi_{w}}{\phi_{b}}\right)
$$

This equation links the wall volume fraction $\phi_{w}$ to the local permeate flux $V_{w}$. To explain the 
limiting flux observed experimentally, Michaels (1968) assumed that the wall concentration cannot exceed a limiting concentration named the gel concentration, $\phi_{g}\left(\phi_{w}\right.$ is replaced by $\phi_{g}$ in Eq. 3). This approach was corroborated by experimental observation of deposits formed on the membrane surface and sometimes recovered after filtration. The value $\phi_{g}$ has to be determined from experimental data, but even if this "gel concentration" is considered to be known, this model for concentration polarization does not satisfactorily predict permeate flux for concentrated solutions and/or for interacting solutes, which colloidal suspensions are by definition.

Some recent work (Bhattacharjee, Kim \& Elimelech, 1999) includes the effects of concentrated and interacting solutes in the convection-diffusion equation (Eq. 2) via a structure factor for interacting particles. This model allows a realistic description of the behavior of concentrated solutions near a membrane, but does not include the transition from concentration polarization to gel or deposit formation. On the other hand, other authors (Benkhala, Ould-Ris, Jaffrin \& Si-Hassen, 1995; Harmant \& Aimar, 1996) give experimental evidence of a TMP beyond which an irreversible deposit is formed by a stable colloid. For given properties of the colloidal dispersion, the deposit appears at a particular permeation flux named the "critical" flux. Deposition is clearly a very important factor in membrane fouling. This critical flux has been shown (Bacchin, Aimar \& Sanchez, 1995; Harmant \& Aimar, 1996) to arise from a balance between the two types of forces acting on a suspended particle near the solid interface (the membrane or the cake):

- the repulsive force due to surface interaction between the colloid and the solid interface driving the particle away from the surface,

- the drag force due to permeate flux drawing the particle towards the surface.

Below the critical flux, the drag force is weaker than the repulsive interaction and no fouling or phase change should occur; above this value, the drag is strong enough to lead to fouling. 
Some recent publications (Bacchin, Aimar \& Sanchez, 1995; Harmant \& Aimar, 1996; Bowen \& Jenner, 1995) stress the importance of colloidal interaction in determining the critical flux but also underline the need to account for the effect of concentration on transport properties.

Now Petsev, Starov \& Ivanov (1993) and Jönsson \& Jönsson (1996) have suggested that the properties of concentrated colloidal dispersions can be described by an expression for the osmotic pressure that takes into account contributions from the entropic effect and colloidal interactions. These authors clearly show that with rising concentration the osmotic pressure theoretically passes through a maximum and that this maximum corresponds to a transition from a dispersed to a solid state. In the case of membrane filtration, this transition may be used in the description of deposit formation, but it has not yet been linked to a critical flux. The main assumption in the model developed by Jönsson \& Jönsson (1996) is that flow occurs through a purely diffusive boundary layer. However the thickness of the boundary layer can only be either roughly estimated from a correlation based on just diffusive mechanisms (a questionable approximation) or else adjusted from experiments.

In the present paper, a model is proposed that introduces colloidal interaction forces into the traditional convective-diffusive mass transfer equation through an interaction term in the osmotic pressure of the suspension. This model is intended to account for the non-ideal behavior of the concentrated suspension or solution in the boundary layer. It predicts realistic properties (diffusion, mobility) of a concentrated colloidal dispersion. In contrast to some previous models, this approach considers mass transfer in two dimensions, thus allowing the growth of concentration polarization, gel layer and deposit along the channel to be described.

\section{THEORETICAL DEVELOPMENT}

We consider the cross-flow filtration of a colloidal dispersion in a tubular configuration at steady state (Figure 1). This system is assumed to have rotational symmetry, thus reducing the three dimensions of the tubular geometry to the two dimensions $r$ and $z$. The hydrodynamics in 
this system will be treated as the sum of tangential shear flow and radial flow. This approximation was shown by Berman (1953) to be acceptable in a thin layer near the permeable wall, when he solved the Navier-Stokes equation in a porous-walled channel with a uniform permeation rate. In the present paper, we consider that the hydrodynamics in the axial direction is not affected by the growth of the cake deposit and that the colloidal matter is totally retained by the membrane, i.e. the pore size is too small to allow particles through. This limits our investigation to surface fouling, including concentration polarization and particle deposition, and excludes pore blocking. Furthermore, we assume the additivity of hydraulic resistance thus limiting the model to deposits with thicknesses greater than several particle diameters. For mass transfer modeling, we consider only interactions between particles: the interaction between the membrane surface and the colloid is not accounted for. This corresponds to neglecting the transient state of membrane/colloid interaction and considering only a balance between suspended and deposited colloid at steady state or considering that the membrane has similar surface properties to those of the particles in suspension. On the other hand, fluid mechanical forces such as shear induced diffusion and lateral migration are not taken into account as they are negligible for particles less than one micrometer in size. The colloid is composed of monodisperse, incompressible and spherical particles or macromolecules in interaction with each other.

\section{Physical representation of the concentrated colloidal dispersion}

For the purpose of our work, the influence of the presence of particles interacting with each other on the rate of water transport at steady state is theoretically accounted for via the osmotic pressure and its variation with concentration. This is then used to derive the diffusivity of the suspended medium that determines the rate of solute transport. We hence adopt the method proposed by Petsev, Starov \& Ivanov (1993).

The diffusion coefficient is derived from the Einstein (1906) equation, which links the 
diffusion coefficient to the derivative of osmotic pressure with respect to volume fraction, together with $V_{p}$ the volume of the suspended particle and its mobility $m$ :

$D(\phi)=m(\phi) V_{p} \frac{d \Pi}{d \phi}$

It should be noted that the diffusion coefficient defined here is not a classical self-diffusion coefficient but is generally termed a gradient-diffusion or collective-diffusion coefficient as it describes the macroscopic flux of particles in the presence of a gradient in the volume fraction of colloidal matter (Russel, Saville \& Schowalter, 1989).

Assuming that the mobility $m(\phi)$ is the reciprocal of the friction factor defined by the Stokes law, as corrected by Happel (1958) to account for the effect of concentration on the drag force, the following expression for the diffusion coefficient is obtained:

$D(\phi)=\frac{V_{p}}{6 \pi \mu a H(\phi)} \frac{d \Pi}{d \phi}$

where $a$ is the particle radius and $\phi$ the volume fraction. The Happel function $H(\phi)$ is given by:

$$
H(\phi)=\frac{6+4 \phi^{\frac{5}{3}}}{6-9 \phi^{\frac{1}{3}}+9 \phi^{\frac{5}{3}}-6 \phi^{2}}
$$

The osmotic pressure is estimated by adding together the contributions of entropy, van der Waals interactions and electrostatic interactions as detailed in the Appendix. It should be noted that the osmotic pressure here is not the total osmotic pressure of the suspension, but the osmotic pressure that originates from the species of the suspension retained by the membrane.

$$
\Pi(\phi)=\Pi_{e n t}(\phi)+\Pi_{v d w}(\phi)+\Pi_{e l e}(\phi)
$$

The magnitude of these different contributions varies with the volume fraction of the suspension, with the particle size and with physico-chemical parameters. An example of the osmotic pressure calculated for suspensions spheres of $5 \mathrm{~nm}$ and $100 \mathrm{~nm}$ in radius is shown in 
Figures 2 \& 3 with each contribution detailed. Other data for calculating osmotic pressure are given in Table 1 (second column). At low volume fraction, the osmotic pressure is dominated by the entropic effect for small spheres (Figure 2) whereas for larger ones (Figure 3) the entropic contribution is negligible and osmotic pressure only results from the electrostatic interaction effect. In both cases for volume fractions higher than a critical value, van der Waals surface interaction becomes predominant and causes a considerable drop in osmotic pressure. As already mentioned by Petsev, Starov \& Ivanov (1993), there thus exists a maximum in theoretical osmotic pressure at a critical concentration denoted $\phi_{c}$. Beyond this critical concentration, attractive interactions lead to an unstable suspension in which the coagulation process can be seen as the result of a "negative diffusion" from dilute (liquid phase) to aggregated (solid phase) suspension. In this work, we consider this critical concentration as the value for the liquid-solid transition, i.e. the transition from concentration polarization to fouling regime. We shall see later in the paper that it probably corresponds to the limiting wall concentration that Michaels (1968) called the "gel" concentration. The dispersion is considered thermodynamically stable towards coagulation at concentrations below $\phi_{c}$, even if a slow coagulation should be expected in real systems at concentrations just below $\phi_{c}$, as pointed out by Jönssson and Jönsson (1996).

Experimentally, the transition between liquid and solid phases appears in a graph of osmotic pressure versus volume fraction as a discontinuity zone indicating the coexistence of both fluid and solid (Russel, Saville \& Schowalter, 1989, p. 342). The transition can also be observed by studying the reversibility of osmotic pressure measurements using the osmotic stress method (Bonnet-Gonnet, Belloni \& Cabane, 1994). It should be noted that the decline in osmotic pressure at volume fractions above the fluid/solid transition (dashed line in Figures $2 \& 3$ ) is not physical and cannot be observed experimentally. In fact, at volume fractions above the maximum a phase transition occurs (often called spinodal decomposition) and a solid is formed : the contact forces between solid surfaces induce an additional resistance to the compression that 
increases with volume fraction, however the latter compression is irreversible whereas osmotic pressure remains reversible. In this work, we use the osmotic pressure only to describe properties of the fluid phase and the maximum in osmotic pressure corresponds to the solid/fluid transition.

This physical description allows the diffusive properties of concentrated interacting particles to be seen as the combination of an entropic effect and colloidal interaction. It should be noted that repulsive colloidal interaction plays a role at intermediate volume fractions by enhancing the gradient diffusion coefficient (as shown in Figure 3 by an increase in osmotic pressure), whereas for high volume fraction attractive interaction reduces diffusivity leading to coagulation beyond $\phi_{c}$. This kind of behavior is qualitatively in agreement with the way that experimental measurements of the osmotic pressure vary with the particle size and the physico-chemical properties of the suspension, such as ionic strength and zeta potential. So even if no fully

predictive theoretical model for concentrated colloidal dispersions exists, the approximate method of simply adding colloidal interaction to the entropic effect provides a good qualitative description of the behavior of a large range of colloidal systems.

\section{Hydrodynamics and mass transfer}

Hydrodynamics and mass transfer in the filtration device (Figure 1) are described from volume (Eq. 8) and mass (Eq. 15) balances in the axial $x$ direction, combined with a convectiondiffusion mass balance in the radial $z$ direction (Eq. 13), including the physical representation of concentrated suspensions as discussed in the previous section.

From a volume balance accounting for permeation, a differential equation is obtained for the axial variation in the total flow rate:

$\frac{d Q}{d x}=-2 \pi R V_{w}$

The axial pressure gradient along the membrane channel is determined by the friction factor for laminar or turbulent flow: 
$\frac{d P}{d x}=P^{\prime}(x)=-f \frac{Q^{2} \rho}{\pi^{2} R^{5}}$

The hydrodynamic regime in the membrane channel depends on the Reynolds number:

$\operatorname{Re}=\frac{2 Q \rho}{\pi R \mu_{b}}$

In the laminar regime $(\operatorname{Re}<2100)$, the friction factor is given by the Poiseuille relationship:

$f=16 / \operatorname{Re}$

whereas for turbulent flow, a common approximation for the friction factor is given by the Blasius correlation:

$f=0.0791 / \operatorname{Re}^{1 / 4}$

Mass transfer in the radial $z$ direction, i.e. perpendicular to the membrane surface, is described by a convection-diffusion equation including the effect of colloidal interaction on the different properties as a function of volume fraction, as developed in the previous section. For a colloid totally retained by the membrane, its comes :

$-V_{w} \phi-D(\phi) \frac{\partial \phi}{\partial z}=0$

As the polarization layer is thin compared with the membrane channel radius, the following equation is valid throughout the polarization layer.

Separation of variables in Eq. 13 allows the coordinate $z$ to be replaced by the concentration $\phi:$

$d z=-\frac{1}{V_{w}} \frac{D(\phi)}{\phi} d \phi$

At steady state, the flux of suspended matter along the channel is constant and equal to the mass flux entering the inlet section of the channel; the left-hand integral in Eq. 15 can be represented as the sum of the mass flux in the bulk (first term) and that in the boundary layer thickness $\delta$ where the concentration polarization is present 


$$
\int_{0}^{R} r u \phi d r=\phi_{b} \frac{Q}{2 \pi}+R \int_{0}^{\delta} u\left(\phi-\phi_{b}\right) d z=\frac{Q_{0} \phi_{b}}{2 \pi}
$$

We adopt the assumption already made by Davis \& Sherwood (1990) and Song \& Elimelech (1995) that there is no variation in bulk concentration in the axial direction of the membrane tube. This assumption is justified by the fact that, in the calculations performed in this paper, the permeate flow rate is always less than one percent of the bulk flow rate $Q$ and the very low diffusivities of colloidal particles imply that concentration variations only appear within the very thin polarisation layer.

Equation (15) can then be rewritten as:

$$
Q=Q_{0}-\frac{2 \pi R}{\phi_{b}} \int_{0}^{\delta} u\left(\phi-\phi_{b}\right) d z
$$

The cross-flow velocity $u$ can be evaluated by assuming that the shear stress throughout the boundary layer thickness is equal to the wall shear stress $R P^{\prime}(x) / 2$ :

$$
u(z)=-\frac{R}{2} P^{\prime}(x) \int_{0}^{z} \frac{d z}{\mu(\phi)}
$$

In this work we have chosen to represent the variation of viscosity with volume fraction by the Eilers-Chong formula (Kissa, 1999):

$$
\frac{\mu(\phi)}{\mu_{b}}=\left[1+\frac{1.25 \phi}{1-\phi / \phi_{c p}}\right]^{2}
$$

where $\mu_{\mathrm{b}}$ is the bulk viscosity and $\phi_{c p}$ is the volume fraction for close packing as defined in the Appendix. In fact $\phi_{c p}$ is likely to be a function of the physico-chemical properties of the system, although its value is much greater than the critical volume fraction that represents the maximum concentration reached in our calculations. Since this work focused on the effect of particles interactions on diffusivity and osmotic pressure, we assumed $\phi_{c p}$ to be a constant: a 
more detailed treatment of this point is left to future work.

By substituting Eqs. 14 and 17 in Eq. 16, we obtain an equation depicting the mass balance with concentration-dependent diffusion and viscosity in the mass-transfer boundary layer :

$$
Q=Q_{0}+\frac{\pi R^{2} P^{\prime}(x)}{\phi_{b} V_{w}^{2}} \int_{\phi_{w}}^{\phi_{b}} D(\phi) \int_{\phi_{w}}^{\phi} \frac{D\left(\phi^{\prime}\right)}{\phi^{\prime} \mu\left(\phi^{\prime}\right)} d \phi^{\prime} d \phi
$$

Using Eq. 9 for the pressure derivative along the membrane and simplifying by introducing a function $G$, Eq. 19 can finally be written:

$$
V_{w}=\frac{Q}{R} \sqrt{\frac{\rho f}{\phi_{b} \pi R} \frac{G\left(\phi_{b}, \phi_{w}\right)}{Q_{0}-Q}}
$$

with $G\left(\phi_{b}, \phi_{w}\right)=\int_{\phi_{b}}^{\phi_{w w}} D(\phi) \int_{\phi}^{\phi_{\phi_{w}}} \frac{D\left(\phi^{\prime}\right)}{\phi^{\prime} \mu\left(\phi^{\prime}\right)} d \phi^{\prime} d \phi$

From considerations of hydrodynamics and mass transfer in the membrane channel, Eq. 20 relates the permeate flux to the volume concentration at the wall. Another relationship linking these two parameters would allow the system to be completely determined. This is achieved here by using the osmotic pressure model to describe the permeate flux through the membrane (cf. Eq. 1):

$V_{w}(x)=\frac{P(x)-\Pi\left(\phi_{w}\right)}{\mu_{0} R_{m}}$

Equations 20 and 22 can be solved simultaneously to give $\phi_{w}$ and $V_{w}$. This non-linear algebraic system can be combined with the ordinary differential equations 8 and 9 so that for given values of pressure $P$ and flow rate $Q$, the gradients $P^{\prime}$ and $Q^{\prime}$ are obtained. This set of firstorder ordinary differential equations can then be solved by a numerical method such as the Runge-Kutta technique.

\section{Critical volume fraction and deposition mechanism}

If $\phi_{w}$ the volume fraction at the wall is less than the critical volume fraction $\phi_{c}$, then only 
concentration polarization is present, with no colloidal deposition on the membrane and the calculation technique outlined above is satisfactory. However, if the calculated $\phi_{w}$ is greater than the critical volume fraction, this implies physically that the high concentration at the membrane has caused a transition of the suspension from a liquid to a solid state, leading to particle deposition. In this case, the permeation equation must include a cake hydraulic resistance $R_{c}$ for this deposit (from Eq. 1):

$V_{w}(x)=\frac{P(x)-\Pi\left(\phi_{c}\right)}{\mu_{0}\left(R_{m}+R_{c}\right)}$

However $R_{c}$ is an unknown value. To solve this problem, we assume that the local cake thickness will increase until a value of $R_{c}$ is obtained such that Eq. 23 will be in agreement with a version of Eq. 20 and 21 in which $\phi_{w}$ is set equal to $\phi_{c}$.

Physically this means that the permeation rate will gradually decline from the value which induced the wall accumulation higher than $\phi_{c}$ until it reaches a value where the diffusionconvection equilibrium at the wall (i.e. at the cake surface) will set $\phi_{w}$ equal to $\phi_{c}$. This procedure of calculation is illustrated in Figure 4. Finally Eq. 23 can be used to calculate the ratio $R_{c} / R_{m}$. The thickness of the cake cannot be calculated, unless its specific hydraulic resistance and density are known, but we shall assume that the cake thickness is negligible when compared to the hydraulic radius of the channel. This assumes that deposit growth does not disturb the axial flow.

\section{DISCUSSION}

Simulations using the model developed in the previous section have been performed for various operating conditions and physico-chemical properties of the media. To illustrate the capability of the model to describe both concentration polarization and particle deposition, simulation results are first presented for two different kinds of colloidal material: small particles (5 $\mathrm{nm}$ in radius) which could represent a macromolecule such as a big protein and larger ones 
(100 nm) like latex particles, for example. The common data set for these simulations is listed in Table 1. These data correspond to colloid ultrafiltration with turbulent cross-flow conditions in a tubular device $(\mathrm{Re}=6000)$. Results of the simulation are the $2 \mathrm{D}$ concentration profile and the local permeate flux along the membrane as illustrated in Figure 5. It shows clearly how the concentration polarization develops at the membrane surface along $x$ the length of the membrane, in a boundary layer whose thickness grows characteristically with $x^{1 / 3}$. Note that wall concentration reaches a value of $\phi \approx 0.5$ at a certain distance from the inlet which will be seen later on in the paper as associated with the formation of irreversible deposit.

\section{Overall analysis of the simulation}

In Figures 6 and 7, the average flux $J$ through the membrane (found by integrating the local flux $V_{w}$ ) for these two suspensions is plotted versus TMP, with other parameter values as in Table 1. Both of these graphs show the typical ultrafiltration behavior, with an initial increase in flux with TMP and at higher TMP, a leveling-off to a quasi-plateau in flux commonly called the "limiting" flux. This "experimenter's view" of the system shows two graphs very similar in shape, but there is an important theoretical difference between them. For the larger colloid (Figure 7), the average flux equals the pure solvent flux right until it reaches the limiting flux. For the macromolecules (Figure 6), the flux is soon well below the water flux and reaches the limiting flux more gradually. This difference can be explained by analyzing the way in which the volume fraction at the interface $\phi_{w}$ and the cake resistance $R_{c}$ (both at the outlet of the membrane channel) vary with TMP, as shown in Figures $6 \mathrm{~b}$ and $7 \mathrm{~b}$.

In the case of macromolecular particles, the increase in concentration polarization with increasing TMP explains the loss in permeate flux without cake formation: the osmotic pressure reaches values comparable with the TMP. As indicated earlier, concentration polarization alone is sufficient to explain the strong limitation in flux. It has to be noted however that for high enough TMP (above $20 \mathrm{kPa}$ in Figure 6), a deposit appears on the membrane even when the flux 
is already clearly limited. The possible link with the appearance of a gel layer is discussed below. In the other case, i.e. for a particulate colloid, the transition is directly correlated to the appearance of a cake resistance at the outlet of the membrane channel. This transition between a no-fouling zone (permeate flux equal to water flux) and the limiting flux (flux invariant with TMP) is very sharp.

These first results show how the model is able to describe the flux reduction due to concentration polarization, to irreversible fouling and to cake formation and the transition between these regimes.

\section{Formation of irreversible fouling: concepts of critical flux and critical Péclet number}

To go further in the analysis, let us take a closer look at the way irreversible fouling can appear locally on the membrane. For this purpose, we define the critical flux as the flux for which an irreversible deposit begins to appear somewhere on the membrane surface, indicated by arrows and the vertical dashed line in Figures 6 and 7. For large and stable colloids, we note by comparing Figures 7a and 7b that the transition between the non-fouling condition and the quasilimiting flux is linked with the occurrence of the critical flux. In this case, the concept of critical flux represents both the first local appearance of irreversible fouling and the average flux above which fouling would be rapid. It should be noted that the average flux may increase beyond the critical flux. This can be analyzed through Figure 9, where the local permeate flux is plotted as a function of $x$, the axial coordinate of the membrane channel, for different TMP's from previous results. As illustrated by the loss in permeate flux, the deposit begins to form near the outlet of the channel, where hydrodynamic conditions are unfavorable and the accumulation is maximum. With increasing TMP, the deposit progressively spreads and finally reaches the entrance of the membrane channel. Gourgues, Aimar \& Sanchez (1992) already suggested this mechanism for cake formation after visual observations of clay deposits on outer-skinned hollow fibers and previous models have predicted this behavior. It should be noted that deposits can be formed 
near the module inlet when there is an important pressure drop along the membrane. An important point of the present model is that particle fouling by a growing cake can be seen as the natural continuation of concentration polarization.

In the case of a macromolecular colloid, i.e. small particles, the flux limitation mainly results from concentration polarization creating an osmotic pressure difference: the flux is limited by osmotic pressure before TMP is high enough for the critical flux to be reached. The local critical flux for a TMP around $20 \mathrm{kPa}$ (black squares in Figure 8) appears all along the membrane at the same TMP. At this point any further increase in pressure will not increase the steady state average flux, but will induce an additional irreversible phase change. In this case, the limiting flux is almost equal to the critical flux value.

As already noted (Bacchin et al., 1995) and confirmed here by simulation, the critical flux is thus a local concept and is reached for different TMP at different positions along the membrane channel. It is therefore more appropriate to introduce a local Péclet number:

$\mathrm{Pe}=\frac{V_{w}(x) \delta(x)}{D_{b}}$

Where $V_{w}$ and $\delta$ are respectively the local permeate flux and boundary layer thickness at a given distance $x$ from the inlet to the membrane channel. The value of the boundary layer thickness is calculated by integration of Eq. 14:

$\delta=\frac{1}{V_{w}} \int_{\phi_{b}}^{\phi_{w}} \frac{D(\phi)}{\phi} d \phi$

The Péclet number represents the ratio of convective transport $V_{w}$ to diffusive transport $D / \delta$ in the boundary layer. The variation of this number along the membrane is represented in Figures 10 and 11 for data corresponding to distribution of permeate flux already plotted in Figures 8 and 9. Note that the distribution of critical flux along the membrane due to hydrodynamics is here represented by a constant Péclet number (the "critical" value $\mathrm{Pe}_{\text {crit }}$ ). For small colloids, in the absence of an irreversible layer, the Péclet number is almost constant along the membrane, 
because of the concentration polarization phenomena which already limit the permeate flux. So the range of TMP for which the irreversible deposit appears over the whole membrane surface is very narrow. The critical value for the Péclet number is reached at different points along the membrane at about the same TMP. This justifies the idea of a critical pressure. On the other hand, in the case of large colloids, in the absence of irreversible fouling, the flux is almost uniform along the membrane surface because of negligible osmotic effects. So the Péclet number increases with $x$ because of the increasing thickness of the boundary layer. Thus the critical Pe is first reached at the outlet of the channel creating a initial unfouled zone followed by a zone with irreversible fouling. The concept of critical Péclet number is of importance for the understanding and the engineering of colloidal filtration as the transition between concentration polarization and cake formation along the membrane surface can be quantified by a single parameter. These considerations must be limited to cases where membrane-suspension and cake-suspension interactions are similar: otherwise there may be one critical Péclet for forming the first monolayer of cake and a different critical Péclet number for cake growth, thus giving more complex behavior during filtration.

To summarize, an important difference between macromolecular and particulate irreversible fouling arises from the way the cake appears in the membrane device:

- in the case of particles ("large" colloids), when the TMP is increased, the cake appears gradually from the outlet to the inlet of the membrane channel leading to a progressive rise in flux from the first local critical flux to the overall limiting flux. On the other hand, the transition between limitation by membrane resistance and limitation by cake formation is very sharp. Thus for a large colloid, cake formation (assimilated in the next section to particle deposition) is a gradually spreading distribution of fouling with a sharp transition from non-fouling to fouling conditions.

- in the case of macromolecules (small colloids) when TMP is increased the phase transition 
appears simultaneously on the whole membrane surface but with a transition between fouling mechanisms smoother than in the case of big particles. The gel layer formation, as defined in next section, is then characterized by a smooth transition between non-limited and limited flux induced by concentration polarization limitation, leading to a simultaneous widespread irreversible fouling.

In this approach, osmotic pressure appears to be a key property. It affects the flux (as traditionally accepted via eq. 1) but it also controls the mass accumulation through the variation in diffusivity (eq. 4). For large particles having little osmotic effect, osmotic pressure still plays an important role as it determine the phase transition and so affects the critical flux for irreversible fouling.

\section{Analysis of transport phenomena implied in the fouling}

The approach adopted in this work underlines important differences in the mechanisms responsible for membrane fouling depending on the size of the colloid filtered. A more detailed analysis of the importance of each transport phenomenon involved in the fouling can be instructive.

Figure 12 summarizes the links between driving forces (permeation, entropic diffusion, attractive and repulsive colloidal interaction) and mechanisms implied in the fouling (concentration polarization, gel formation and deposition). First, transport phenomena can be classified into three categories:

- the driving phenomenon for fouling is permeation. At the same time, it is essential for the separation and can be seen as the separation force. This antagonism explains the fact that pressure driven membrane separation can never be performed without mass accumulation.

- the regulating phenomenon, such as entropic diffusion or repulsive colloidal interaction, limits fouling. These phenomena can be seen globally as mixing effects, in contrast with the demixing (separating) effect of permeation. 
- the irreversible phenomenon, attractive interaction, emphasizes fouling and introduces an irreversible character. This third category of transport phenomena is responsible for the liquid/solid transition leading to the deposition of particles and can be seen as a "reaction" from liquid to solid phase.

Conditions for fouling are the result of a physical combination of these transport phenomena. Concentration polarization is a totally reversible mass accumulation at the membrane surface. This mechanism implies a balance between a driving and a regulating phenomenon and does not bring into play attractive interactions between the solute in solution and the solute already deposited. The regulating phenomenon for small particles can be entropic diffusion which is associated with an osmotic pressure comparable to the driving TMP. In this case, concentration polarization reduces permeate flux below the pure water flux (see Figure 6, below the critical flux). For large colloids or very concentrated macromolecular solutions, concentration polarization is a balance between permeation and repulsive interaction. But the osmotic pressure here is very low and filtration below the limiting flux is essentially limited by the membrane resistance. To summarize, for large colloids, concentration polarization does occur but it has hardly any effect on filtration because of the low value of osmotic pressure.

If the colloidal system exhibits attractive interaction for small inter-particle distances (i.e. high volume fraction) and if the driving force is high enough, surface attraction between solute in solution and solute already deposited leads to an irreversible fouling phase (liquid to solid transition). At this point, two cases can be considered depending on the nature of regulating phenomenon:

- If repulsive interaction is dominant (large colloids - right-hand side of Figure 12), the high concentration of repulsive colloid at the membrane surface should lead to an orderly organization of particles in the polarized layer according to Russel, Saville \& Schowalter (1989). The liquid/solid transition would occurs if the volume fraction exceeds the critical 
value, would then create a relatively compact and organized deposit at the membrane surface. One might imagine this as a "ballistic" deposition mechanism with contacts resulting from linear trajectories (Chapter 6.4 in Elimelech, Gregory, Jia \& Williams, 1995); these linear trajectories would arise from the oriented nature of diffusion in ordered suspensions (collective diffusion).

- In the opposite case, the importance of entropic diffusion will create a disordered solid phase formed under totally attractive interaction. Each contact between solute in suspension and solute deposited on the membrane will result in a liquid/solid transition. Moreover, because of Brownian diffusion, contacts will occur in all directions. The result is a "dendritic" layer leading to a loose deposit, usually called a gel. It is clear that between these extreme cases there can exist situations intermediate between gel and deposit, depending on the physicochemical properties of the filtered media.

Figure 12 brings these ideas together in a simple form. It can be seen as an operating diagram where fouling mechanisms are located as a function of the driving force and the colloid size (and/or intensity of surface interaction), where four areas appear: concentration polarization, gel layer, non-fouling and deposition (Figure 13). Globally, the driving force can, if high enough, give rise to irreversible phenomena. The colloid size (or more generally its physico-chemical properties) plays a role in the way the deposit is formed at the membrane surface (gel formation and deposition). The transition between reversible and irreversible fouling and its effect on flux is increasingly sharper as the colloid size increases (as illustrated by the gray gradation in Figure 13). With the engineering aspect in mind, it would be interesting to determine exact transitions between these fouling mechanisms whose consequences for the process are radically different. For example from Figure 6, one has to limit the TMP to $20 \mathrm{kPa}$ to prevent the formation of a strongly irreversible gel layer. Such a transition depends on physico-chemical properties such as ionic strength or zeta potential. It is then possible to determine a critical Péclet number to 
characterize the transition between non-fouling and deposition. Our objective in future work will be to use the model to delimit quantitatively the border shown in Figure 13 as a function of the physico-chemical properties of the suspension and operating conditions.

\section{Limiting and critical flux}

The limiting flux is the maximum stationary flux obtained when increasing TMP. As discussed in the previous section, consideration of the transport phenomena involved in fouling makes it possible to distinguish two kinds of limiting fluxes: limiting flux controlled by entropic diffusion (LFED) and limiting flux controlled by surface repulsion (LFSR). Unlike the limiting flux, the critical flux is a criterion for the transition between concentration polarization and fouling, i.e. when attractive interaction occurs between colloids near the membrane (critical flux for surface attraction CFSA). The critical flux is reached when irreversible fouling occurs at a certain point on the membrane, whereas the limiting flux is reached when the whole membrane surface operates at the critical flux. Experimentally, the critical flux can only be measured by having each step up in TMP followed by a smaller step down so as to detect the first irreversibility in the system; in comparison, the limiting flux is easy to measure. But the critical flux can provide more information on fouling and on the way the process has to be run. Whereas solute accumulation can never be totally eliminated from a filtration process, irreversible fouling can be avoided if the process is run below the critical flux. This possibility opens up interesting perspectives for the filtration of stable colloids exhibiting low osmotic pressure.

\section{CONCLUSIONS}

The model presented and discussed in this paper is capable of accounting for concentration polarization, gel-layer formation and particle deposition and depicting the continuity between the major fouling mechanisms involved in ultrafiltration of colloidal suspensions. It shows that a single theoretical approach is capable of covering a wide range of suspension sizes and of cross 
flow situations. Basic transport phenomena, such as convection and diffusion (with colloidal interactions and their concentration dependence taken into account) are sufficient to create a realistic description of concentrated suspensions without introducing any adjustable parameters, such as an a priori value for the gel concentration. In the present model, colloidal interaction acts both on transport phenomena (diffusive properties of the suspension) and on the interface process of cake formation. This complexity of colloidal suspensions allows a physically realistic description of mass accumulation (by separation and mixing in a boundary layer) and liquid/solid transition (by "reaction" at the membrane surface). In particular, the variation of osmotic pressure with the volume fraction of the suspension seems to be a key element for the fouling model, as it specifies the condition for liquid/solid transition, i.e. the formation of a gel layer or deposit. This two-dimensional simulation allows realistic suspension physics to be combined with an accurate representation of hydrodynamics.

Through simulation, the local permeate flux and the 2D concentration distribution may be visualized. Variation of stationary flux with transmembrane pressure (TMP) clarifies the contribution of membrane resistance, concentration polarization and irreversible layer formation to limiting the flux for different sizes of colloids. The relative importance of these different effects is found to depend on the operating conditions (such as TMP) and the suspension properties (colloid size and physico-chemical parameters). The model shows its capacity to describe the continuous transition from concentration polarization to cake formation by seeing cake formation in a feed-back relationship with the concentration polarization: the cake layer is formed when the volume fraction at the membrane exceeds a critical value corresponding to a maximum in osmotic pressure and the cake formation causes a decline in flux until concentration returns to the critical value. Physically, the attractive colloidal interaction between particles (cause of the drop in osmotic pressure at high concentration) is shown to be responsible for the coagulation of particles on the membrane giving an irreversible solid layer. This irreversible 
layer is seen to appear in the membrane device for a critical Péclet number which is constant along the membrane surface, as this dimensionless number takes into account the difference in hydrodynamic conditions. It thus appears as a key parameter for engineering the process. The following distinction is made between two mechanisms for the formation of irreversible layers:

- The gelation mechanism: formation of loose layer with a low fractal dimension, appearing simultaneously across the membrane surface.

- The deposition mechanism: formation of a compact ordered deposit growing progressively from the outlet to the inlet of the membrane device.

From the numerical tools provided by the model and the better understanding of fouling that it allows, tools for process optimization must now developed. As an example, precise determination of the border between fouling mechanisms can help in choosing operating conditions within the reversible accumulation zone. Here the critical Péclet number is obviously a key parameter for engineering the process. On the other hand, our speculations about gel or deposit formation need to be checked experimentally and could open up interesting perspectives for understanding the structure of deposit layers.

\section{ACKNOWLEDGMENTS}

The authors are grateful to Indo-French Centre for the Promotion of Advanced Research (grant $\left.n^{\circ} 1615-1\right)$ for supporting a part of this work (D.S.H.).

\section{APPENDIX}

The osmotic pressure of a suspension of interacting colloidal particles is considered as the sum of entropic, electrostatic and van der Waals contributions, which are evaluated by using a cell model.

The entropic contribution due to the particle distribution is approximated by Hall's (1972) equation for hard spheres: 
$\Pi_{\text {ent }}(\phi)=\frac{3 k T}{4 \pi a^{3}} \phi \frac{\left(1+\phi+\phi^{2}-0.67825 \phi^{3}-\phi^{4}-0.5 \phi^{5}-X \phi^{6}\right)}{1-3 \phi+3 \phi^{2}-1.04305 \phi^{3}}$

with $\left.X=6.2028 \exp \mid\left(\phi_{c p}-\phi\right)\left\{7.9-3.9\left(\phi_{c p}-\phi\right)\right\}\right]$

and $\phi_{\mathrm{cp}}=\frac{\pi \sqrt{2}}{6}$ as in hexagonal close packing.

In the low-concentration limit, the traditional van't Hoff equation is obtained.

The van der Waals contribution is obtained by differentiating the van der Waals free energy with respect to the number of solvent molecules; this gives (Jönsson \& Jönsson, 1996):

$\Pi_{v d w}(\phi)=-\frac{z_{n} A}{48 \pi a^{3}} \frac{\phi^{3}}{\left(\phi_{c p}-\phi_{c p}^{1 / 3} \phi^{2 / 3}\right)^{2}}$

where $z_{n}$ is the number of neighboring particles in the cell lattice (12 in the case of a hexagonal lattice) and $A$ is the Hamaker constant.

When interacting particles are charged, electrostatic interactions occurs between particles. Their contribution is included in the osmotic pressure calculation using the Wigner-Seitz cell approach and solution of the Poisson-Boltzmann equation. Bowen \& Jenner (1995) derive the following relationship:

$\Pi_{\text {elec }}(\phi)=\frac{\varepsilon}{z_{n}} N_{A} k T c_{0}\left[\cosh \left(\frac{\kappa a \zeta^{*}}{\kappa a \phi^{-1 / 3} \cosh \left\{\kappa a\left(1-\phi^{-1 / 3}\right)\right\}+\sinh \left\{\kappa a\left(1-\phi^{-1 / 3}\right)\right\}}\right)-1\right]$

where $\zeta^{*}=e z \zeta / k T$ is the reduced zeta potential, $\kappa$ the Debye-Hückel parameter, $N_{A}$ Avogadro's number and $I$ the ionic strength.

\section{REFERENCES}

Aimar P. \& Field R.W. (1992). Limiting flux in membrane separations: a model based on the viscosity dependency of the mass transfer coefficient. Chem. Eng. Sci., 47, 579-586.

Altena F. W. \& Belfort G. (1984). Lateral migration of spherical particles in porous flow channels: application to membrane filtration. Chem. Eng. Sci., 39 (2), 343. 
Bacchin P., Aimar P. \& Sanchez V. (1995). Model for colloidal fouling of membranes, AIChE J., 41 (2) 368-376.

Berman A.S. (1953). J. Appl. Phys., 24, 1232.

Benkhala Y.K., Ould-Ris A., Jaffrin M.Y. \& Si-Hassen D. (1995). Cake growth mechanism in cross-flow microfiltration of mineral suspensions. J. Membrane Sci., 98, 107-117.

Bhattacharjee S., Kim A.S. \& Elimelech M. (1999). Concentration polarization of interacting solute particles in cross flow membrane filtration. J. Colloid Interface Sci, 212, 81-99.

Bonnet-Gonnet C., Belloni L. \& Cabane B. (1994), Osmotic pressure of latex dispersions Langmuir, 10, 11, 4012-4021.

Bowen W. R. \& Jenner F. (1995). Dynamic ultrafiltration model for charged colloidal dispersions : a Wigner-Seitz cell approach. Chem. Eng. Sci., 50 (11), 1707-1736

Cohen R.D. \& Probstein R.F. (1986). Colloidal fouling of reverse osmosis membranes. $J$. Colloid Interface Sci., 114 (1).

Davis R. H.\& Sherwood J. D. (1990) A similarity solution for steady-state crossflow microfiltration. Chem. Eng. Sci., 45 (11), 3203-3209.

Einstein A. (1956). Investigation on the theory of the brownian movement, ed. R. Furth, New York : Dover Publications.

Elimelech M., Gregory J., Jia X. \& Williams R. (1995). Particle deposition \& aggregation: Measurement, modeling and simulation. Oxford : Butterworth-Heinemann.

Gourgues C., Aimar P. \& Sanchez V. (1992). Ultrafiltration of bentonite suspensions with hollow fiber membrane. J. Membrane Sci., 74, 51-69.

Hall K.R. (1972). Another hard sphere equation of state. J. Chem. Phys., 57, 2252-4.

Happel J. (1958). Viscous flow in multiparticle systems, slow motion of fluids relative to beds of spherical particles. AIChE J., 4 (2), 197.

Harmant P. \& Aimar P. (1996). Coagulation of colloids retained by porous wall. AIChE J., 42 
(12), 3523-3532 .

Howell J.A. (1995). Subcritical flux operation of microfiltration. J. Membrane Sci., 107, 165171.

Jönsson A.S. \& Jönsson B. (1996). Ultrafiltration of colloidal dispersions - A theoretical model of the concentration polarization phenomena. J. Colloid Interface Sci., 180, 504-518.

Kissa E. (1999). Dispersions: characterization, testing and measurement, Marcel Dekker, New York, N.Y.

McDonogh R.M., Fane A.G. \& Fell C.J.D. (1989). Charge effects in the cross flow filtration of colloids and particulates. J. Membrane Sci., 43, 69-85.

Petsev D.N., Starov V.M. \& Ivanov I.B. (1993) Concentrated dispersions of charged colloidal particles: sedimentation, ultrafiltration and diffusion. Colloids and Surfaces A, 81, 65-81.

Russel W. B., Saville D.A. \& Schowalter W.R. (1989). Colloidal dispersions. Cambridge : Cambridge Univ. Press.

Song L. \& Elimelech M. (1995). Theory of concentration polarization in cross-flow filtration. J. Chem. Soc. Faraday Trans., 91, 3389-3398.

Zydney A.L. \& Colton C.K. (1986). A concentration polarization model for the filtrate flux in cross flow microfiltration of particulate suspensions. Chem. Eng. Comm., 47, 1-21.

\section{NOMENCLATURE}
A Hamaker constant, M.L ${ }^{2} \cdot \mathrm{T}^{-2}$
a particles or macromolecules radius; $\mathrm{L}$
D diffusion coefficient, $\mathrm{L}^{2} \cdot \mathrm{T}^{-1}$
f friction factor
H Happel correction for sedimentation velocity
J Mean permeate flux, L.T $T^{-1}$
k Boltzman constant, M.L ${ }^{2} \cdot \mathrm{T}^{-2} \cdot \mathrm{K}^{-1}$ 
P pressure, $M \cdot L^{-1} \cdot T^{-2}$

Q flow rate, $\mathrm{L}^{3} \cdot \mathrm{T}^{-1}$

$\mathrm{R}$ membrane channel radius, $\mathrm{L}$

m mobility of particle or macromolecule, $\mathrm{M}^{-1}$.T

$\mathrm{R}_{\mathrm{c}}$ hydraulic resistivity of deposit, $\mathrm{L}^{-1}$

$R_{m}$ hydraulic resistivity of membrane, $L^{-1}$

$\mathrm{T}$ temperature, $\mathrm{K}$

u axial velocity, $\mathrm{L} . \mathrm{T}^{-1}$

$\mathrm{v}_{\mathrm{w}}$ local permeate flux at the wall, $\mathrm{L} \cdot \mathrm{T}^{-1}$

Vp Particle or macromolecule volume, $\mathrm{L}^{3}$

$\mathrm{x}$ axial direction in the membrane channel, $\mathrm{L}$

$\mathrm{z}$ radial direction in the membrane channel, $\mathrm{L}$

\section{Subscript}

0 inlet

b bulk

c critical

сp close packed

w wall

\section{Greek letters}

$\delta \quad$ diffusive boundary layer thickness, L

$\phi \quad$ volume fraction

$\kappa \quad$ inverse of Debye length, $\mathrm{L}^{-1}$

$\Pi \quad$ osmotic pressure, $M \cdot \mathrm{L}^{-1} \cdot \mathrm{T}^{-2}$

$\rho$ solution density, $M . L^{-3}$

$\mu \quad$ viscosity, $M \cdot L^{-1} \cdot T^{-1}$

$\zeta \quad$ zeta potential, $\mathrm{V}$ 


\section{FIGURE CAPTIONS}

Figure 1. Major parameters and co-ordinates in cross-flow filtration in a cylindrical channel.

Figure 2. Variation of the total osmotic pressure with the volume fraction. ( $a=5 \mathrm{~nm}$, other data as in Table 1).

Figure 3. Variation of the total osmotic pressure with the volume fraction. ( $a=100 \mathrm{~nm}$, other data as in Table 1).

Figure 4. Calculation procedure with a criterion for distinguishing cake formation from concentration polarization.

Figure 5. 2D concentration profile for $100 \mathrm{~nm}$ suspension and TMP $=15 \mathrm{kPa}$ (others conditions as in Table 1). The corresponding permeate flux profile is presented in Figure 9.

Figure 6. Simulation of membrane fouling for interacting suspension of $5 \mathrm{~nm}$ radius: (a) mean permeate flux as a function of the transmembrane pressure, (b) volume fraction at the membrane and cake hydraulic resistance at the channel outlet as a function of TMP.

Figure 7. Simulation of membrane fouling for interacting suspension of $100 \mathrm{~nm}$ radius: (a) Mean permeate flux as a function of the transmembrane pressure (b) Volume fraction at the membrane and cake hydraulic resistance at the membrane outlet plotted as a function of TMP.

Figure 8. Permeate flux profile along the dimensionless length of the membrane channel for 5 nm suspension. Profiles are shown for different transmembrane pressures.

Figure 9. Permeate flux profile along the dimensionless length of the membrane channel for $100 \mathrm{~nm}$ suspension.

Figure 10. Variation of Péclet number along the dimensionless length of the membrane channel for $5 \mathrm{~nm}$ suspension. Profiles are shown for different transmembrane pressures.

Figure 11. Variation of Péclet number along the dimensionless length of the membrane channel for $100 \mathrm{~nm}$ suspension. 
Figure 12. Transport phenomena (bold characters) involved in membrane fouling and mechanisms (underlined characters) coupled together by transport phenomena.

Figure 13. Diagram showing fouling mechanisms as a function of colloid size (or surface repulsion) and driving force (transmembrane pressure or permeate flux).

\section{TABLES}

Table 1. Common data set used for simulations 
fig.1

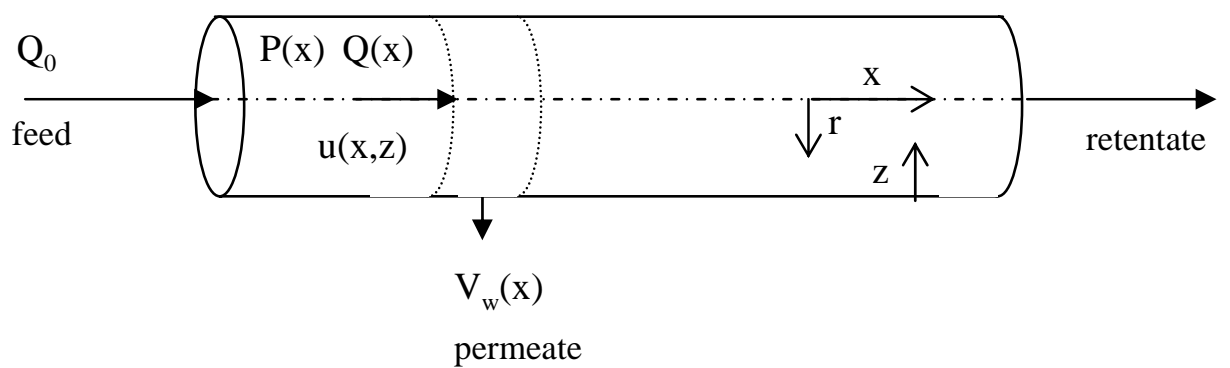


fig.2

transition

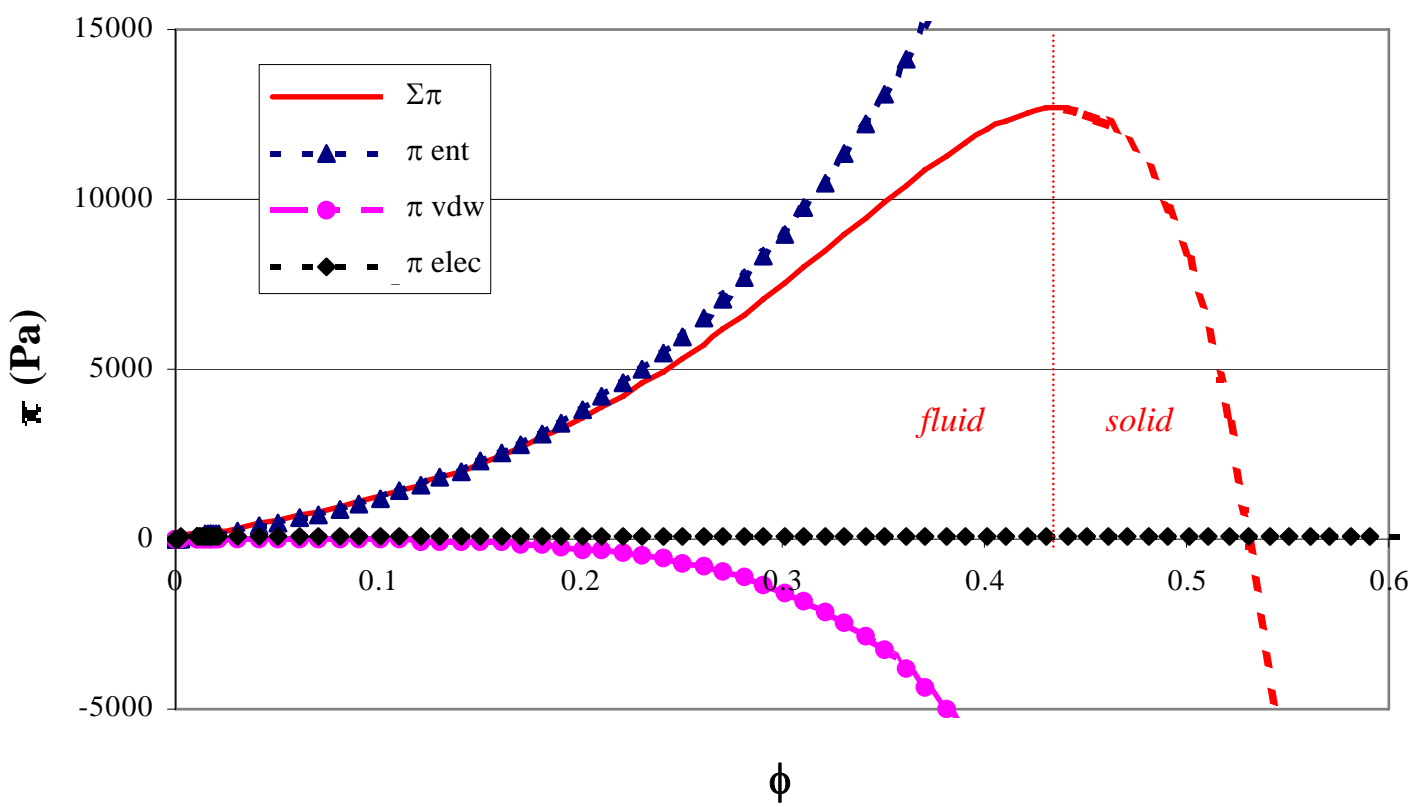


fig.3

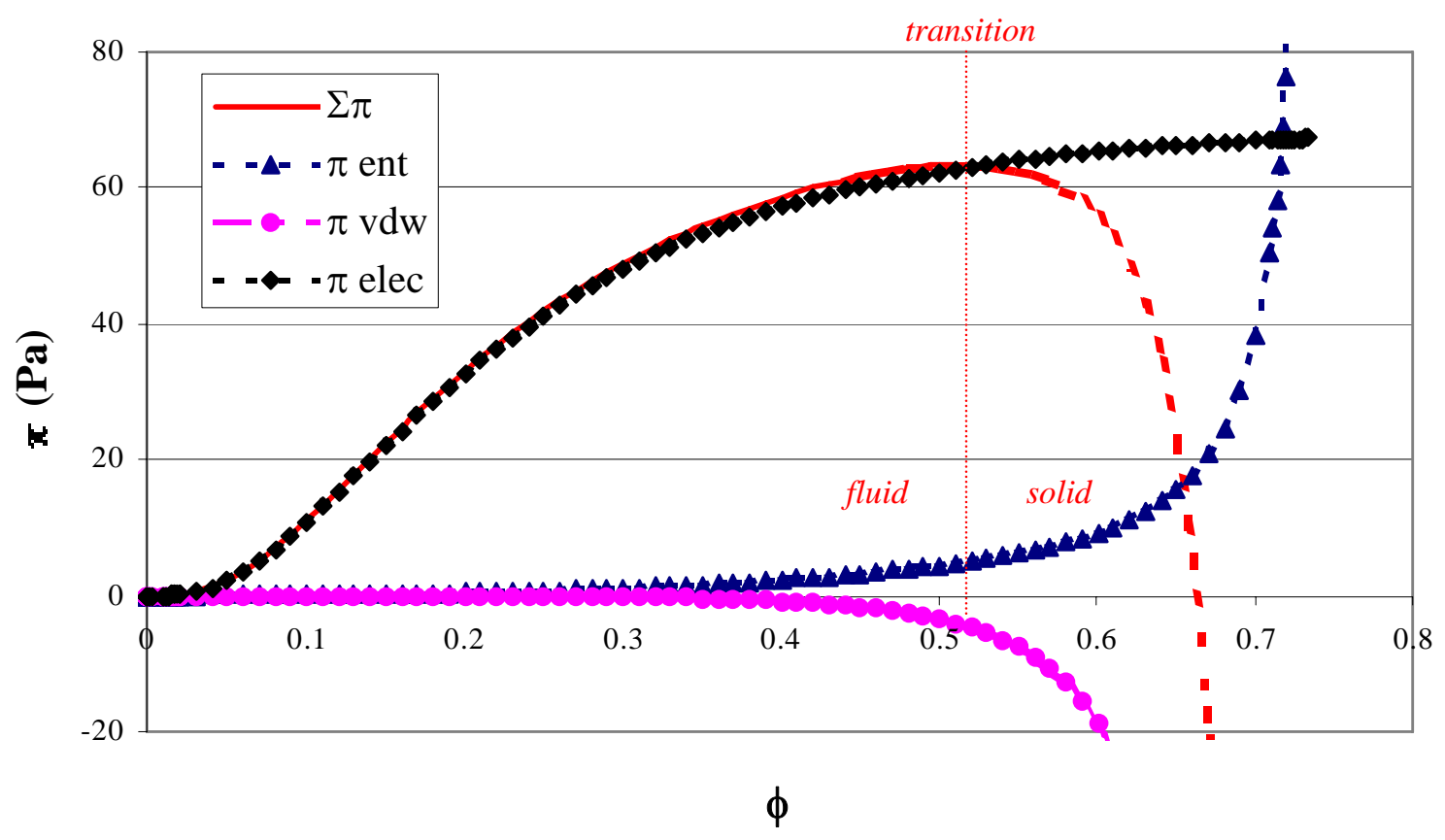


fig.4

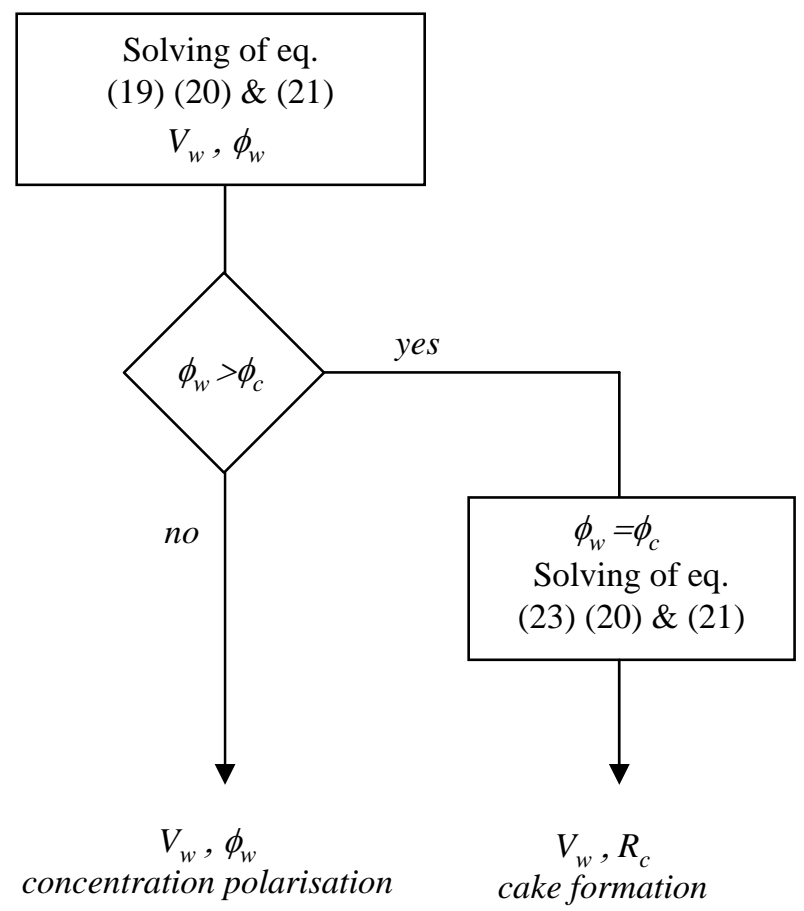


fig.5

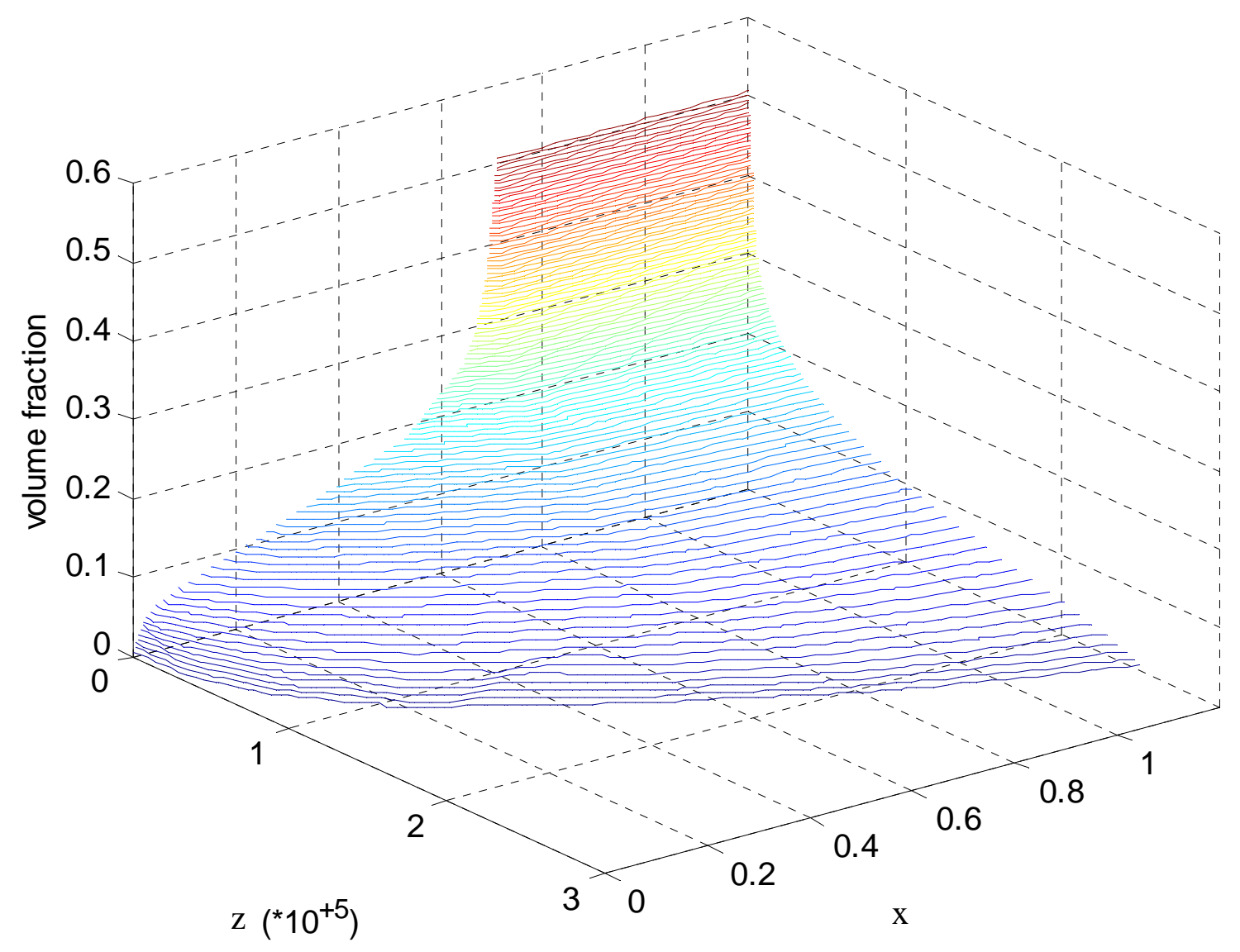


fig.6

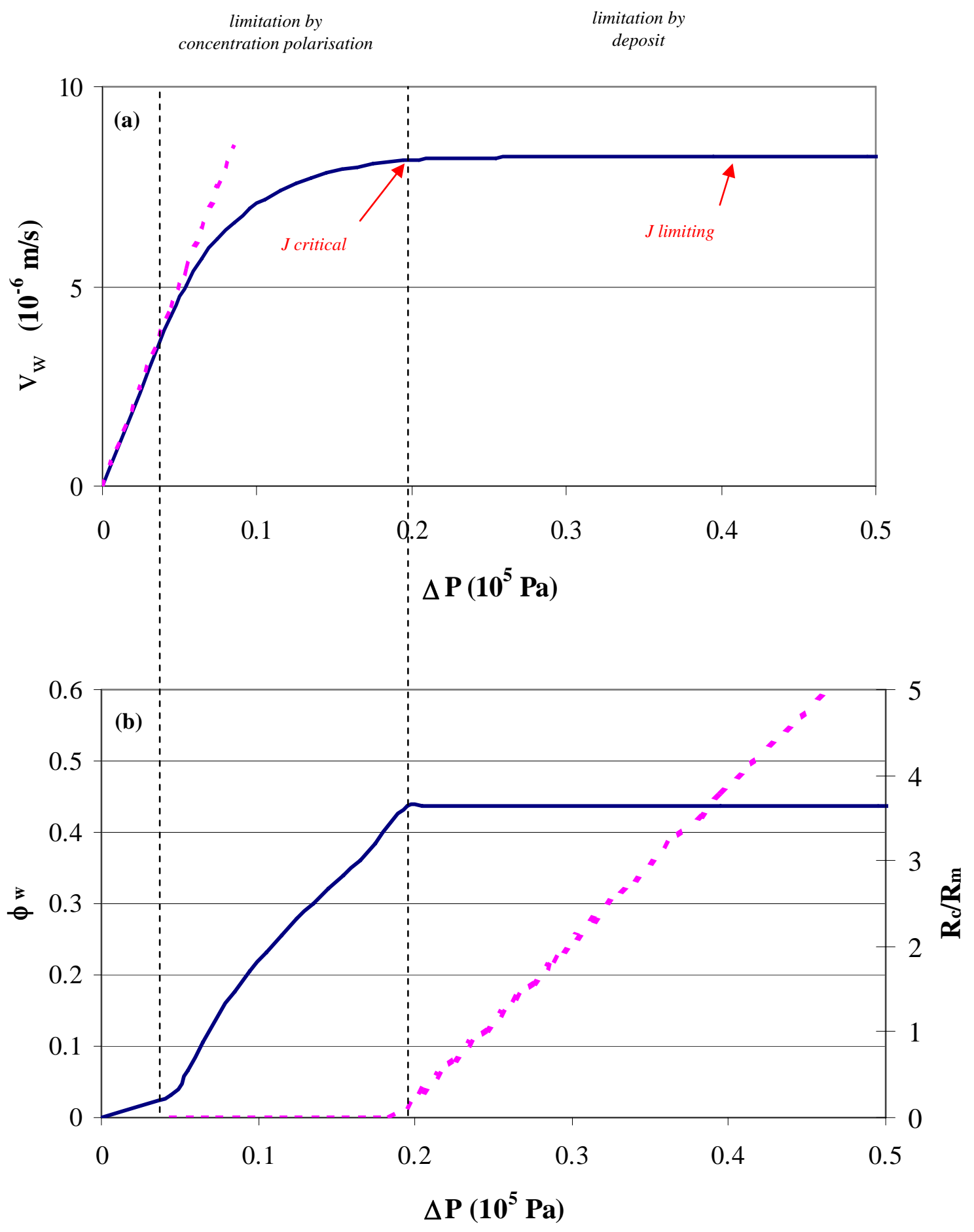


fig.7

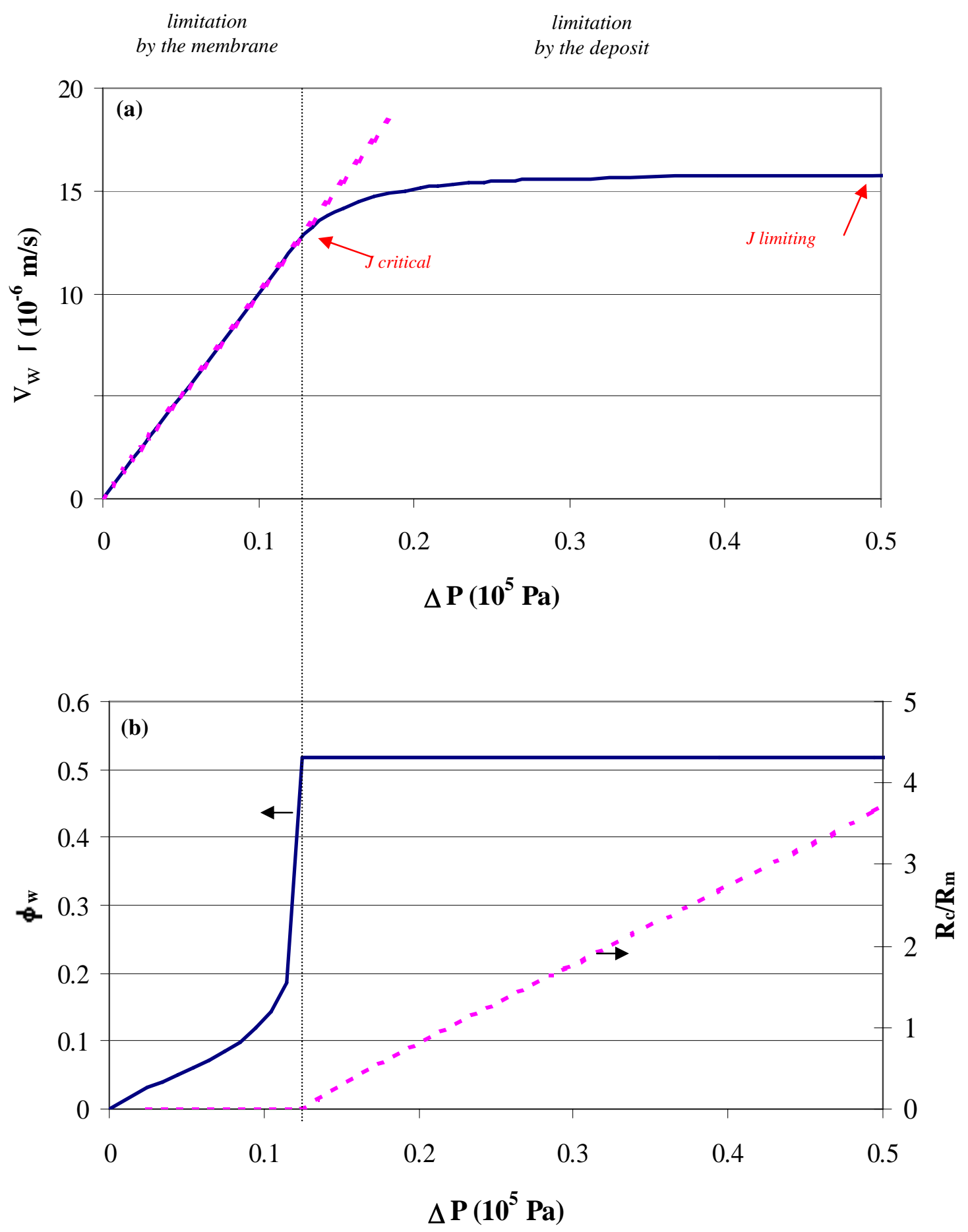


fig.8

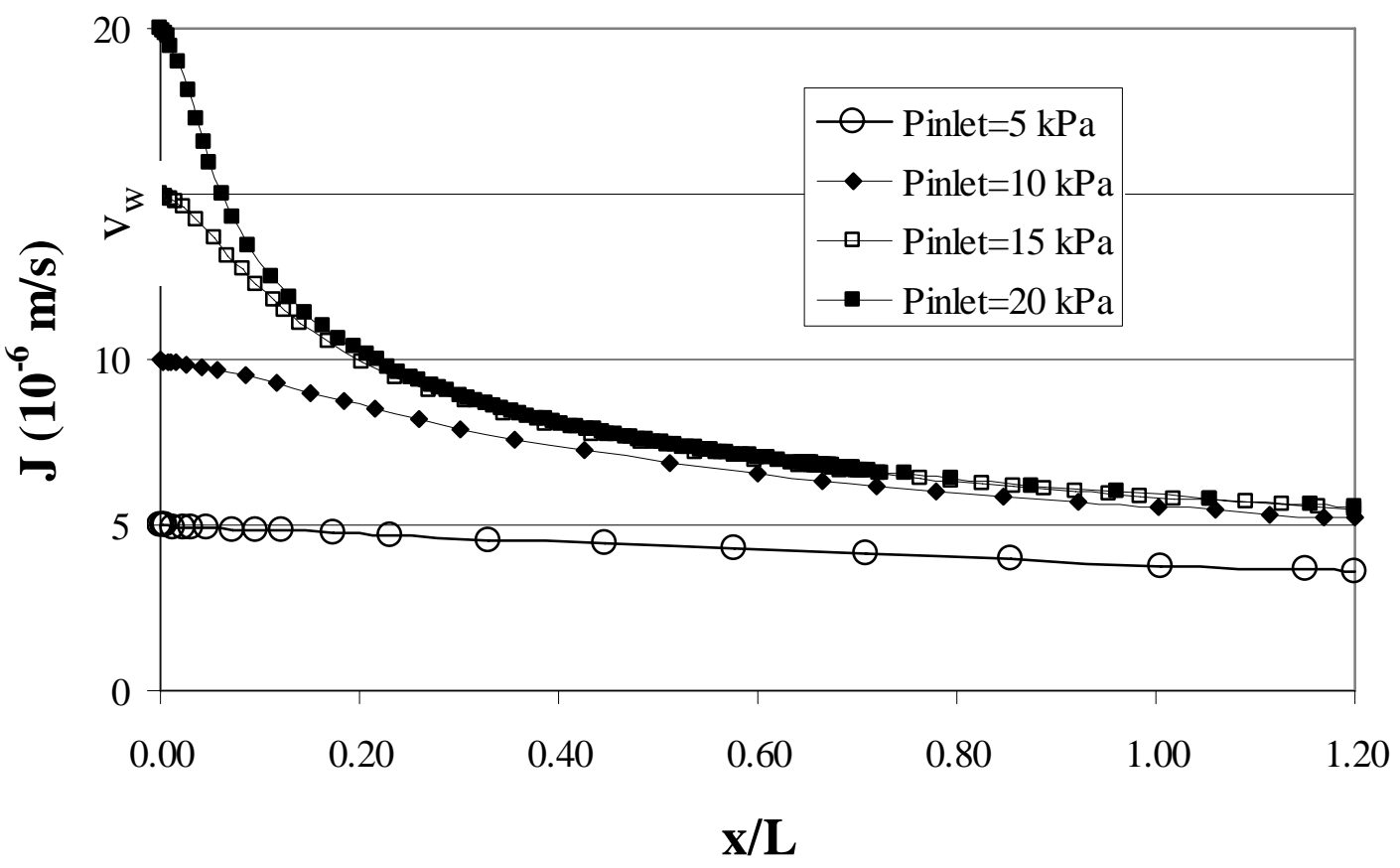


fig.9

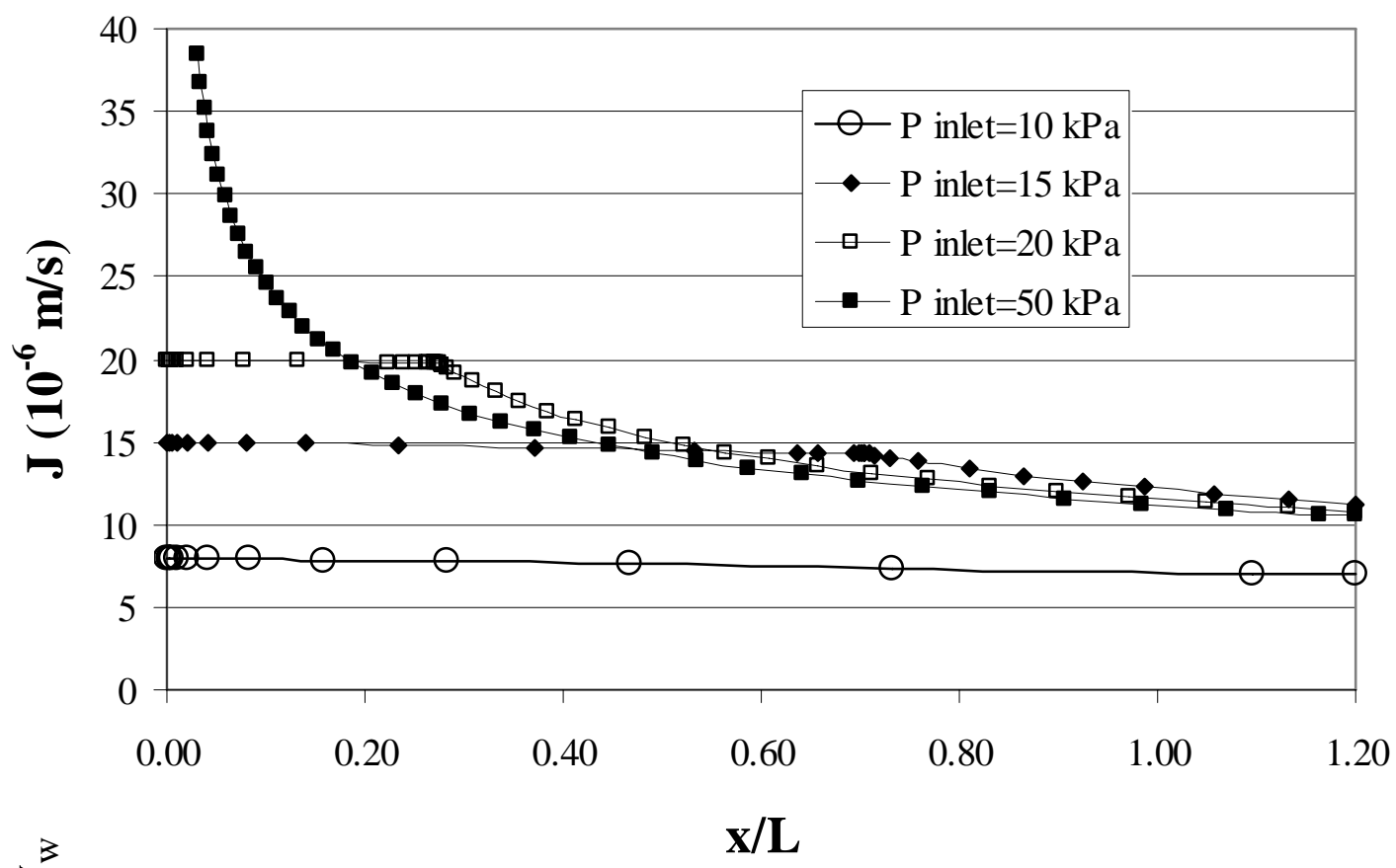


fig.10

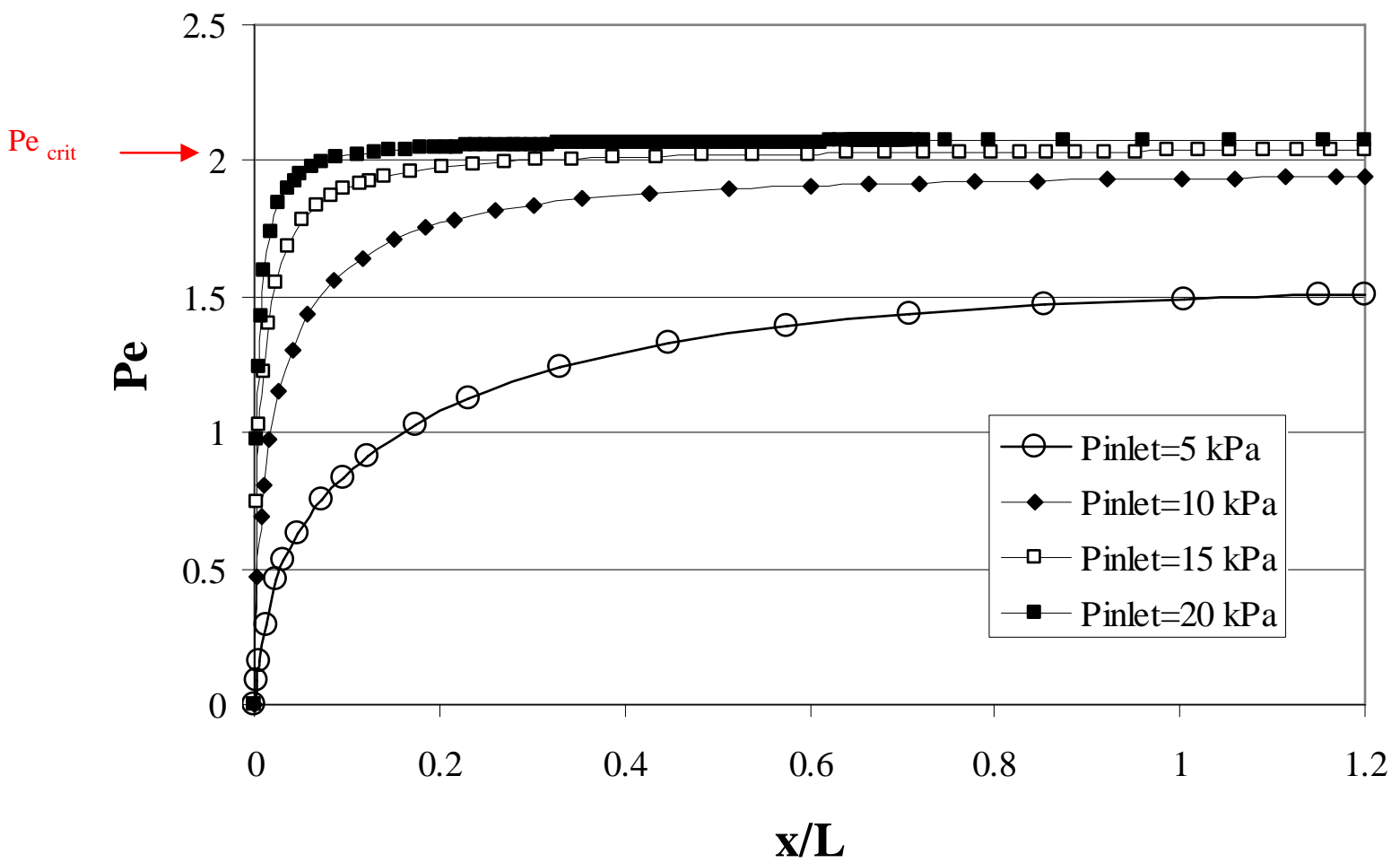


fig.11

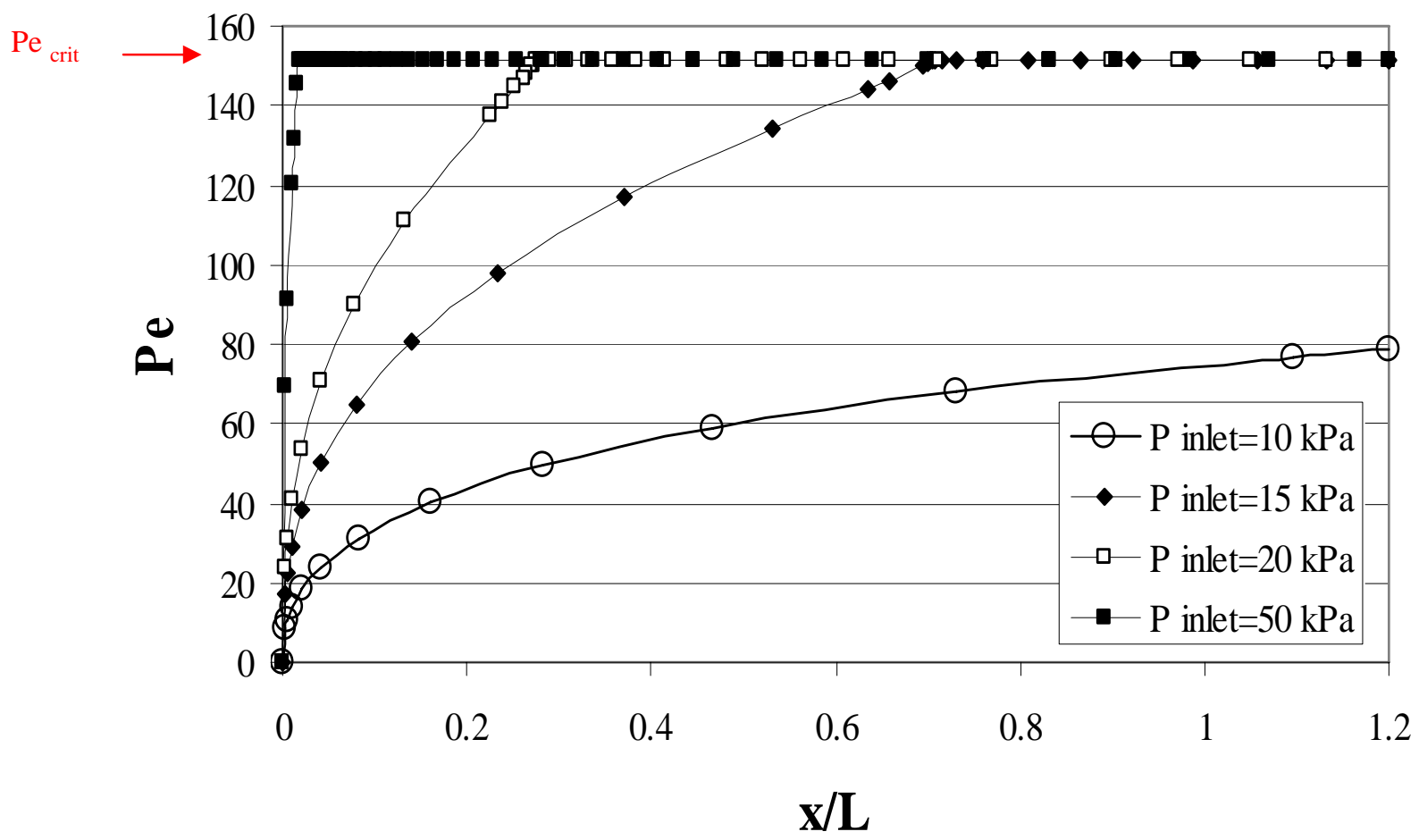


fig.12

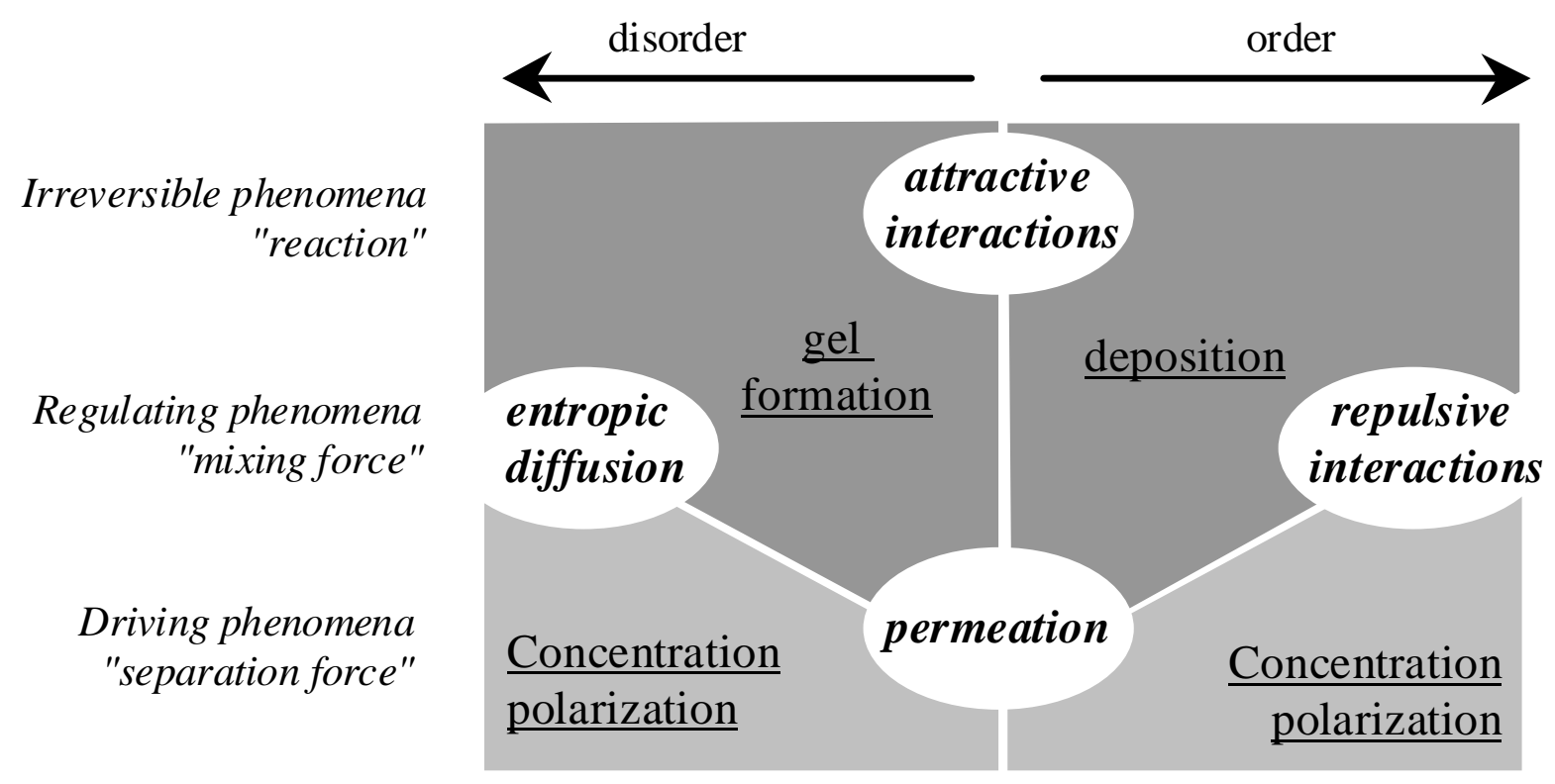


fig.13

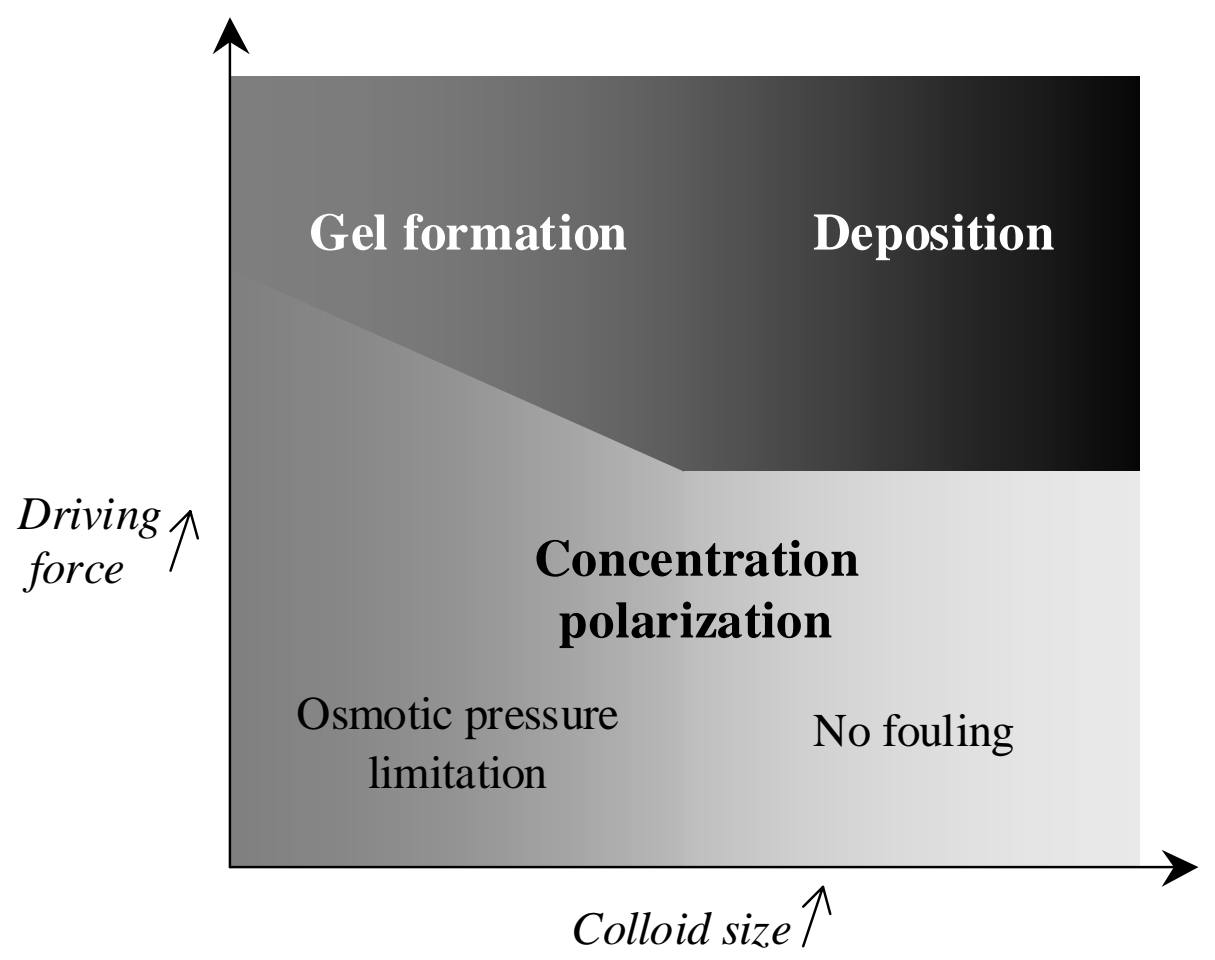

